Bulletin of the Section of Logic

Volume 46:1/2 (2017), pp. 33-45

http://dx.doi.org/10.18778/0138-0680.46.1.2.04

Alexander S. Karpenko

\title{
FOUR-VALUED LOGICS BD and DM4: EXPANSIONS
}

\begin{abstract}
The paper discusses functional properties of some four-valued logics which are the expansions of four-valued Belnap's logic DM4. At first, we consider the logics with two designated values, and then logics defined by matrices having the same underlying algebra, but with a different choice of designated values, i.e. with one designated value. In the preceding literature both approaches were developed independently. Moreover, we present the lattices of the functional expansions of DM4.
\end{abstract}

Keywords: Belnap's four-valued logic, expansions and functional properties, lattices.

\section{With two designated values}

The majority of contemporary works, devoted to Belnap's four-valued logic and its extensions, put forward problems related to the intuitive meaning of connectives and the appropriate interpretation of truth-values. In contrast, we are interested in comparing the functional properties of various fourvalued logics. Therefore, the main tool for us will be the concept of the logical matrix $\mathfrak{M}=\langle A, D\rangle$, where $A$ is a universal algebra and $D$ is a set of designated elements. The detailed theory of logical matrices is investigated in the book by Malinowski [24].

In [8] De and Omori consider an axiomatic expansion BD+ of fourvalued Belnap-Dunn logic by classical negation $\neg$. 
The logical matrix for $\mathbf{B D}$ is the following:

$$
\mathfrak{M}_{\mathrm{BD}}=\langle\{\mathbf{t}, \mathbf{b}, \mathbf{n}, \mathbf{f}\}, \sim, \wedge, \vee,\{\mathbf{t}, \mathbf{b}\}\rangle, \text { where }
$$

\begin{tabular}{|c|c|}
\hline$x$ & $\sim x$ \\
\hline $\mathbf{t}$ & $\mathbf{f}$ \\
$\mathbf{b}$ & $\mathbf{b}$ \\
$\mathbf{n}$ & $\mathbf{n}$ \\
$\mathbf{f}$ & $\mathbf{t}$ \\
\hline
\end{tabular}

\begin{tabular}{|c|cccc|}
\hline$\wedge$ & $\mathbf{t}$ & $\mathbf{b}$ & $\mathbf{n}$ & $\mathbf{f}$ \\
\hline $\mathbf{t}$ & $\mathbf{t}$ & $\mathbf{b}$ & $\mathbf{n}$ & $\mathbf{f}$ \\
$\mathbf{b}$ & $\mathbf{b}$ & $\mathbf{b}$ & $\mathbf{f}$ & $\mathbf{f}$ \\
$\mathbf{n}$ & $\mathbf{n}$ & $\mathbf{f}$ & $\mathbf{n}$ & $\mathbf{f}$ \\
$\mathbf{f}$ & $\mathbf{f}$ & $\mathbf{f}$ & $\mathbf{f}$ & $\mathbf{f}$ \\
\hline
\end{tabular}

\begin{tabular}{|c|cccc|}
\hline$V$ & $\mathbf{t}$ & $\mathbf{b}$ & $\mathbf{n}$ & $\mathbf{f}$ \\
\hline $\mathbf{t}$ & $\mathbf{t}$ & $\mathbf{t}$ & $\mathbf{t}$ & $\mathbf{t}$ \\
$\mathbf{b}$ & $\mathbf{t}$ & $\mathbf{b}$ & $\mathbf{t}$ & $\mathbf{b}$ \\
$\mathbf{n}$ & $\mathbf{t}$ & $\mathbf{t}$ & $\mathbf{n}$ & $\mathbf{n}$ \\
$\mathbf{f}$ & $\mathbf{t}$ & $\mathbf{b}$ & $\mathbf{n}$ & $\mathbf{f}$ \\
\hline
\end{tabular}

Here negation $\sim$ is called 'paraconsistent negation'1.

The truth table for classical (Boolean) negation $\neg$ is given by

\begin{tabular}{|c|c|}
\hline$x$ & $\neg x$ \\
\hline $\mathbf{t}$ & $\mathbf{f}$ \\
$\mathbf{b}$ & $\mathbf{n}$ \\
$\mathbf{n}$ & $\mathbf{b}$ \\
$\mathbf{f}$ & $\mathbf{t}$ \\
\hline
\end{tabular}

In $\mathfrak{M}_{\mathrm{BD}+}$ the classical implication $x \rightarrow y$ is definable by $\neg x \vee y$. In [8] De and Omori present a Hilbert style system BD+ in the propositional language $\{\sim, \wedge, \vee, \neg, \rightarrow\}^{2}$ with modus ponens as the single inference rule (16 axiom schemata).

They also compare $\mathbf{B D}+$ to some related systems found in the literature. In [5] Beziau considers a four-valued modal logic PM4N with the matrix

$$
\mathfrak{M}_{\mathbf{P M} 4 \mathbf{N}}=\langle\{\mathbf{t}, \mathbf{b}, \mathbf{n}, \mathbf{f}\}, \neg, \wedge, \vee, \square,\{\mathbf{t}, \mathbf{b}\}\rangle,
$$

where $\square$ obeys the following truth table:

\begin{tabular}{|c|c|}
\hline$x$ & $\square x$ \\
\hline $\mathbf{t}$ & $\mathbf{t}$ \\
$\mathbf{b}$ & $\mathbf{f}$ \\
$\mathbf{n}$ & $\mathbf{f}$ \\
$\mathbf{f}$ & $\mathbf{f}$ \\
\hline
\end{tabular}

${ }^{1}$ Usually this negation is referred to as De Morgan negation.

${ }^{2}$ Logical connectives and matrix operations will be denoted in the same way. 
In [8] the authors proved (Theorem 5) that PM4N and $\mathbf{B D}+$ are (functionally) equivalent, i.e.

in $\mathfrak{M}_{\mathrm{PM} 4 \mathbf{N}} \quad x \rightarrow y=\neg x \vee y$, and $\sim x=\neg x \leftrightarrow(\square x \vee \square \neg x)$;

in $\mathfrak{M}_{\mathrm{BD}+} \square x=x \wedge(x \leftrightarrow \neg \sim x)$, where in both cases $x \leftrightarrow y=$ $(x \rightarrow y) \wedge(y \rightarrow x)$.

Let's pay attention to the logic BDe from [8] with language $\left\{\sim, \wedge, \vee, \neg^{e}\right\}$, where $\neg^{e}$ (exclusion negation) obeys the following truth table:

\begin{tabular}{|c|c|}
\hline$x$ & $\neg^{e} x$ \\
\hline $\mathbf{t}$ & $\mathbf{f}$ \\
$\mathbf{b}$ & $\mathbf{f}$ \\
$\mathbf{n}$ & $\mathbf{t}$ \\
$\mathbf{f}$ & $\mathbf{t}$ \\
\hline
\end{tabular}

The axiomatization of $\mathbf{B D e}$ is obtained through a minor modification of $\mathrm{BD}+$.

In the next section we will consider logics BD+, PM4N and BDe.

\section{With one designated value}

Belnap's four-valued logic over the language $\{\sim, \wedge, \vee\}$ appears in [4] as semantically defined entailment relation between sentences. Here Belnap also described the history of four-valued truth-tables for $\sim, \wedge$ and $\vee$ in connection with 'logic of first-degree entailments'. Note that in [26] Pietz and Rivieccio considers Belnap's logic with only one designated value.

There are many works about the connection between Belnap's logic and the class of De Morgan lattices ${ }^{3}$. A fundamental work is Font [13] where Belnap's four-valued logic was studied from the algebraic point of view. The main result is that the class of De Morgan lattices is the algebraic

\footnotetext{
${ }^{3}$ De Morgan lattice is a distributive lattice $\langle A, \wedge, \vee\rangle$ with the operation $\sim$. The unary operation $\sim$ satisfies the following equations: $\sim \sim x=x, \sim(x \wedge y)=\sim x \vee \sim y$, $\sim(x \vee y)=\sim x \wedge \sim y$. De Morgan lattices were introduced in 1935 by G. Moisil. The theory of De Morgan lattices is very similar to that of De Morgan algebras (bounded De Morgan lattices) which were investigated in [6] under the name quasi-Boolean algebras. In this work the four-element De Morgan lattice with operations $\{\wedge, \vee, \sim\}$ was firstly considered.
} 
counterpart of Belnap's logic. So some authors denote Belnap's logic as DM4.

Of special interest is Ermolaeva and Mučnik's paper [9] which considers an expansion of De Morgan algebras by Boolean negation $\neg$. The resulting algebras are called $M B$-algebras ${ }^{4}$. The completeness of the axioms of $M B$ algebras and topological representation of $M B$-algebras are proved. Note that operations $\neg$ and $\sim$ commute among themselves, i.e.: $\neg \sim(x)=$ $\sim \neg(x)$. A new operation is denoted by $g(x)$ and it obeys the following truth table:

\begin{tabular}{|c|c|}
\hline$x$ & $g(x)$ \\
\hline $\mathbf{t}$ & $\mathbf{t}$ \\
$\mathbf{b}$ & $\mathbf{n}$ \\
$\mathbf{n}$ & $\mathbf{b}$ \\
$\mathbf{f}$ & $\mathbf{f}$ \\
\hline
\end{tabular}

In [12] $g(x)$ is called 'conflation'.

Note that in $M B$-algebras modal operations are definable:

$$
\begin{aligned}
& \square x=x \wedge g(x), \\
& \diamond x=\sim \square \sim x=x \vee g(x) .
\end{aligned}
$$

The question arises: what logic is determined by the four-element $M B$ algebra with modal operations and one designated value? The answer is as follows: it is an expansion of Lewis modal logic S5, i.e. S5 plus

$$
\square A \vee \square(A \rightarrow B) \vee \square(A \rightarrow \neg B)[32, \text { p. 305 }]^{5} .
$$

It was remarked in $[9$, p. 190] that the four-valued matrix of "group III" from [22, p. 493], in our denotations the matrix

$$
\mathfrak{M}_{\mathrm{V} \mathbf{2}}=\langle\{\mathbf{t}, \mathbf{b}, \mathbf{n}, \mathbf{f}\}, \rightarrow, \neg, \square,\{\mathbf{t}\}\rangle,
$$

is characteristic for $\mathbf{V} 2$. Note that the matrix $\mathfrak{M}_{\mathbf{C}}=\langle\{\mathbf{t}, \mathbf{b}, \mathbf{n}, \mathbf{f}\}, \rightarrow$, $\neg,\{\mathbf{t}\}\rangle$ is characteristic for classical propositional logic $\mathbf{C 2}$.

\footnotetext{
${ }^{4}$ It is interesting that Pynko [28] introduces a similar algebraic structure called $D e$ Morgan boolean algebra. He also suggests Gentzen-style axiomatization of four-valued logic denoted by DMB4.

${ }^{5}$ In [33] this logic is denoted by V2.
} 
It is evident that Béziau's four-valued modal logic PM4N and the logic V2 are functionally equivalent.

In [10] Ermolaeva and Mučnik introduced $B g$-algebras (Boolean algebras with endomorphism $g$ ) and proved Stone's representation theorem for them. They remarked that $B g$-algebra with involution, where $g g(x)=x$, corresponds to the logic V2. And they showed that in matrix for V2 operation $g(x)$ is definable by $\square x \vee(\neg x \wedge \triangleright x)^{6}$. We denote the four-valued logic over the language $\{\neg, \wedge, \vee, g\}$ as $\operatorname{Tr}$. About this logic see below.

Now we return to the logic BDe from [8], but with one designated value.

The expansion of DM4 by the endomorphism $e_{2}$ :

\begin{tabular}{|c|c|}
\hline$x$ & $e_{2}(x)$ \\
\hline $\mathbf{t}$ & $\mathbf{t}$ \\
$\mathbf{b}$ & $\mathbf{t}$ \\
$\mathbf{n}$ & $\mathbf{f}$ \\
$\mathbf{f}$ & $\mathbf{f}$ \\
\hline
\end{tabular}

leads to the logic which G.H. von Wright, in 1985, denoted as $\mathbf{T}^{\prime \prime} \mathbf{L M}$ and called truth-logic (see [35]). For the sake of brevity, we will denote it as $\mathbf{T}^{\prime \prime}$. Here a truth-operator $T$ is the endomorphism $e_{2}$.

It remains to add that the logics $\mathbf{T}^{\prime \prime}$ and $\mathbf{B D e}$ are functionally equivalent, since $\neg^{e}(x)=\sim e_{2}(x)$ and $e_{2}(x)=\sim \neg^{e}(x)$.

Note that the following definitions hold:

$$
e_{1}(x)=\sim\left(e_{2}(\sim x)\right) \text { and } e_{2}(x)=\sim\left(e_{1}(\sim x)\right) .
$$

It is important that all four-valued $J_{i}(x)$-operations (introduced in [29]) are definable in $\mathbf{T}^{\prime \prime}$, where

$$
J_{i}(x)=\left\{\begin{array}{l}
\mathbf{t}, \text { if } x=i \\
\mathbf{f}, \text { if } x \neq i .
\end{array} \quad(i=\mathbf{t}, \mathbf{n}, \mathbf{b}, \mathbf{f})\right.
$$

\footnotetext{
${ }^{6}$ However, see [31, p. 49], where this formula appears for the first time.
} 
Thus, we have:

\begin{tabular}{|c|c|c|c|c|}
\hline$x$ & $J_{\mathrm{t}}(x)$ & $J_{\mathrm{b}}(x)$ & $J_{\mathrm{n}}(x)$ & $J_{\mathrm{f}}(x)$ \\
\hline $\mathbf{t}$ & $\mathbf{t}$ & $\mathbf{f}$ & $\mathbf{f}$ & $\mathbf{f}$ \\
$\mathbf{b}$ & $\mathbf{f}$ & $\mathbf{t}$ & $\mathbf{f}$ & $\mathbf{f}$ \\
$\mathbf{n}$ & $\mathbf{f}$ & $\mathbf{f}$ & $\mathbf{t}$ & $\mathbf{f}$ \\
$\mathbf{f}$ & $\mathbf{f}$ & $\mathbf{f}$ & $\mathbf{f}$ & $\mathbf{t}$ \\
\hline
\end{tabular}

One may easily verify that

$$
\begin{aligned}
& J_{\mathrm{t}}=e_{1}(x) \wedge e_{2}(x), \\
& J_{\mathrm{b}}=\sim e_{1}(x) \wedge e_{2}(x), \\
& J_{\mathrm{n}}=e_{1}(x) \wedge \sim e_{2}(x), \\
& J_{\mathrm{f}}=\sim e_{1}(x) \wedge \sim e_{2}(x)(\text { see }[17, \text { p. } 42]) .
\end{aligned}
$$

Note that $e_{2}(x)=J_{\mathrm{t}} \vee J_{\mathrm{b}}$. Then Wright's logic $\mathbf{T}^{\prime \prime}$ is De Morgan logic DM4 with all $J_{i}(x)$-operations.

Now we need some additional definitions. A finite-valued logic $L_{n}$ with all $J_{i}(x)$-operations is called truth-complete logic, and a logic $L_{n}$ is said to be $\mathbf{C}$-extending iff in $L_{n}$ one can functionally express: the binary operations of implication, disjunction, conjunction, and the unary negation operation, whose restrictions to the subset $\{0,1\}$ coincide with the classical logical operations of implication, disjunction, conjunction, and negation. In virtue of the result of [2], every truth-complete and $\mathbf{C}$-extending logic has Hilbert-style axiomatization extending the $\mathbf{C}_{\mathbf{2}}$. It means that Wright's $\mathbf{T}^{\prime \prime}$ logic has such an axiomatization. Moreover, it follows from [1] that we have an adequate first-order axiomatization for logic $\mathbf{T}^{\prime \prime}$ with quantifiers.

At last, in [8] Corollary 17 asserts that $\mathbf{B D}+$ is not functionally complete. We can give a more precise description of functional properties of $\mathrm{BD}+$.

Let $P_{4}$ be Post's four-valued functionally complete logic (see [27]). The set of operations $R$ is called functionally precomplete in $P_{4}$ if every enlargement $\{R, f\}(=R \cup\{f\})$ of the set $R$ by an operation $f$ such that $f \notin R$ and $f \in P_{4}$ is functionally complete (in other terminology, a precomplete class of operations is called maximal clone). 
Let us consider the set of operations $\left\{\sim, \wedge, \vee, \neg, \neg^{e}\right\}$. Since here we have all $J_{i}(x)$-operations (see above) we can define $x \cup y=\max (x, y)$, $x \cap y=\min (x, y)$ :

$$
\begin{aligned}
x & \cup y=(x \wedge y) \vee\left(J_{\mathrm{f}}(x) \wedge y\right) \vee\left(x \wedge J_{\mathrm{f}}(y)\right) \vee\left(J_{\mathrm{b}}(x) \wedge y\right) \vee\left(x \wedge J_{\mathrm{b}}(y)\right) \vee \\
J_{\mathrm{t}}(x) & \vee J_{\mathrm{t}}(y), \\
x & \cap y=\neg(\neg x \cup \neg y) .
\end{aligned}
$$

In 1941 Moisil introduced $n$-valued Łukasiewicz algebras, but in 1956 A. Rose showed for $n \geq 5$ that it is not possible to define the $n$-valued Łukasiewicz implication in terms of the primitive operations considered by Moisil (see [7]). For us, it means that four-valued Łukasiewicz implication $\rightarrow_{\mathrm{E}}$

\begin{tabular}{|c|cccc|}
\hline$\rightarrow_{\mathrm{t}}$ & $\mathbf{t}$ & $\mathbf{b}$ & $\mathbf{n}$ & $\mathbf{f}$ \\
\hline $\mathbf{t}$ & $\mathbf{t}$ & $\mathbf{b}$ & $\mathbf{n}$ & $\mathbf{f}$ \\
$\mathbf{b}$ & $\mathbf{t}$ & $\mathbf{t}$ & $\mathbf{b}$ & $\mathbf{n}$ \\
$\mathbf{n}$ & $\mathbf{t}$ & $\mathbf{t}$ & $\mathbf{t}$ & $\mathbf{b}$ \\
$\mathbf{f}$ & $\mathbf{t}$ & $\mathbf{t}$ & $\mathbf{t}$ & $\mathbf{t}$ \\
\hline
\end{tabular}

is definable in matrix

$$
\mathfrak{M}_{\mathrm{L}}=\left\langle\{\mathbf{t}, \mathbf{b}, \mathbf{n}, \mathbf{f}\}, \neg, \cup, \cap, J_{\mathrm{t}}, J_{\mathrm{b}}, J_{\mathrm{n}}, J_{\mathrm{f}},\{\mathbf{t}\}\right\rangle .
$$

We can do it in the following way:

$x \oplus y=\left(x \cup \neg\left(J_{\mathrm{f}}(y)\right) \cap\left(y \cup \neg\left(J_{\mathrm{f}}(x)\right) \cap\left(\neg x \cup \neg y \cup J_{\mathrm{t}}(x) \cup J_{\mathrm{b}}(x) \cup J_{\mathrm{t}}(y) \cup\right.\right.\right.$ $\left.J_{\mathrm{b}}(y)\right)$

(cf. Iorgulescu [15, p. 168]),

$x \rightarrow_{\mathrm{E}} y=\neg x \oplus y$.

Primitive operations of Łukasiewicz logic $\mathbf{E}_{4}$ are exactly $\neg$ and $\rightarrow_{\mathrm{E}}$ (in our denotations). ${ }^{7}$ Note that class operations from $E_{4}$, corresponding to $\mathbf{E}_{4}$, preserve truth-values $\mathbf{t}$ and $\mathbf{f}$. It follows from [16] that a given class of operations is precomplete in $P_{4}$. It means that the addition, to this class of the operation which does not preserve $\mathbf{t}$ and $\mathbf{f}$, turns it in a functionally complete class.

${ }^{7}$ The Łukasiewicz $n$-valued logics $(n \geq 3)$ were introduced in 1922 (see the historical note of Malinowski in [34]). 


\section{Lattices of functional expansions of DM4}

So, we have the following lattice of expansions of $D M 4$ :

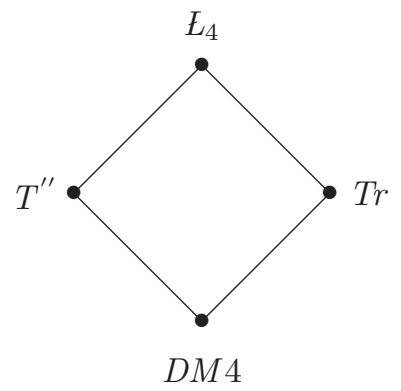

Fig. 1

Note that $\{\neg, \wedge, \vee, g\}(=\operatorname{Tr})$ and $\{\sim, \wedge, \vee, g\}$ are functionally equivalent:

$$
\sim x=\neg g(x) \text { and } \neg x=\sim g(x) .
$$

HYPOTHESIS. The class operations $T^{\prime \prime}$ and $T r$ are functionally precomplete in $\epsilon_{4}$.

In other terminology, $T^{\prime \prime}$ and $T r$ are submaximal clones (about submaximal clones see in [19].

Omori and Sano (see [25]) represented the expansions of $B D$ in the form of Hasse diagram. Here $B D \triangle$ is $T^{\prime \prime}$ and $B D-$ is $T r$, where $\triangle$ is $e_{2}$ and - is $g$. Then $B D \triangle$ and $B D-$ are extended to the functionally complete logic of Post $P_{4} .^{8}$

Now, we will represent a lattice of expansions of $B D$ by endomorphisms $g, e_{1}$ and $e_{2}$ (in distributive lattices) and by constants $b$ and $n .^{9}$ Together with identity operation $e_{o}(x)=x$, operations $g, e_{1}$ and $e_{2}$ form a monoid of all endomorphisms of DM4.

${ }^{8}$ It is worth noting, that quite numerous works deal with functional extensions of $B D$ to $P_{4}$. One of the earliest on the subject is Ruet's paper [30]. There, author uses the 'quarter turn' function - the latter being nothing else but cyclical negation in $P_{4}[27]-$ in order to extend $B D$ to $P_{4}$. See also Arieli and Avron [3] and Pynko [28].

${ }^{9}$ The same (a lattice of expansions) for four-element Boolean algebra was made in [11]. 
Let's consider the following closed classes of operations from $P_{4}$ :

$$
\begin{aligned}
& D M 4=(\sim, \vee, \wedge) ; \operatorname{Tr}=(\vee, \wedge, \sim, g) ; \\
& T^{\prime \prime}=\left(\sim, \vee, \wedge, e_{1}\right)=\left(\vee, \wedge, \sim, e_{2}\right) ; \\
& D M 4 \mathbf{b}=(\sim, \vee, \wedge, \mathbf{b}) ; D M 4 \mathbf{n}=(\sim, \vee, \wedge, \mathbf{n}) ; \\
& T^{\prime \prime} \mathbf{b}=\left(\sim, \vee, \wedge, e_{1}, e_{2}, \mathbf{b}\right) ; T^{\prime \prime} \mathbf{n}=\left(\sim, \vee, \wedge, e_{1}, e_{2}, \mathbf{n}\right) ; \\
& D M 4 \mathbf{b}, \mathbf{n}=(\sim, \vee, \wedge, \mathbf{b}, \mathbf{n}) .
\end{aligned}
$$

Let's show that $\left(\vee, \wedge, \sim, e_{1}, e_{2}, \mathbf{b}, \mathbf{n}\right)=P_{4}$ :

$$
\begin{aligned}
& g(x)=\left(\mathbf{n} \wedge e_{1}(x)\right) \vee\left(\mathbf{b} \wedge e_{2}(x)\right)(\text { see }[11, \text { p. 302] }), \\
& \neg(x)=g(\sim(x)) .
\end{aligned}
$$

We already know that $\left(\sim, \vee, \wedge, e_{1}, e_{2}, \neg\right)=E_{4}$.

Since $E_{4}$ is precomplete (see above) in $P_{4}$ and $E_{4}$ preserve $\mathbf{t}$ and $\mathbf{f}$, then $\left(\sim, \vee, \wedge, e_{1}, e_{2}, \mathbf{b}, \mathbf{n}\right)=P_{4}$.

Lattice of classes given above is shown in Fig. 2:

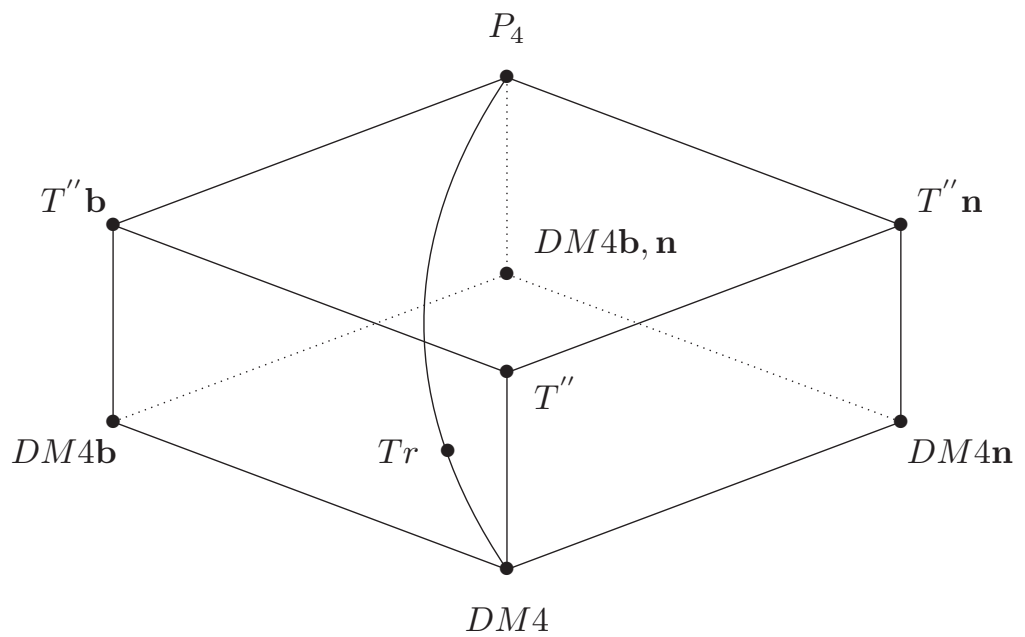

Fig. 2 


\section{Logic $\mathrm{Tr}$}

A very simple axiomatization of truth logic $\operatorname{Tr}$ over the language $\{\rightarrow, \neg, T\}$ with one designated value, where the truth operation $T$ (modality) is the endomorphism $g$, was suggested in $[18]^{10}$ :

(A0) The set of all propositional tautologies (including formulas with modal operation $T$ ).

(A1) $T(A \rightarrow B) \leftrightarrow(T A \rightarrow T B)$.

(A2) $\neg T A \leftrightarrow T \neg A$.

(A3) $T T A \leftrightarrow A$.

The rules of inference: modus ponens and Gödel's rule for $T$.

Let's consider logic Tr with the axiom:

(A4) $T A \leftrightarrow A$.

We denote this logic by $\operatorname{Tr}^{c}$. If we take the operation $T$ as identity operation of $\mathbf{C}_{2}$, then the logic $\operatorname{Tr}^{\mathbf{c}}$ is a conservative extension of $\mathbf{C}_{2}$.

Note than in [21, section $\mathrm{V}]$ the Kripke frame, consisting of two possible worlds, is presented for V2. In [23] Maksimova considers all normal extensions of modal logic $\mathbf{S} \mathbf{4}$ with the Craig interpolation property. From this it follows that modal logic V2 is the single normal extension of modal logic S5 with the Craig interpolation property (between $\mathbf{S 5}$ and $\mathbf{C}_{2}$ ). Since the logics $\operatorname{Tr}$ and V2 are functionally equivalent then the following theorem takes place:

Theorem 1. A logic $\boldsymbol{T r}$ has the Craig interpolation property.

It is worth mentioning that there is a generalized truth-value space in form of a bilattice (see [14]). Indeed, the simplest bilattice is just the four-valued Belnap's logic. In [12] Fitting extends a first-order language by notation for elementary arithmetic, and builds the theory of truth based on a bilattice. This four-valued theory of truth is an alternative to Tarski's approach. Also in one case, Fitting extends this language by the operation conflation (endomorphism $g$ ).

\footnotetext{
${ }^{10}$ In [20] the completeness of logic $\operatorname{Tr}$ is proved with use of Sahlqwist's powerful theorem, which gives the sufficient condition of Kripke completeness for normal modal logic. Algebraic completeness of logic Tr is also proved.
} 


\section{References}

[1] O. M. Anshakov and S. V. Rychkov, On the axiomatization of finite-valued logical calculi, Math. USSR Sbornik 51 (1985), pp. 473-491.

[2] O. M. Anshakov and S. V. Rychkov, On finite-valued propositional logical calculi, Notre Dame Journal of Formal Logic 36, 4 (1995), pp. 606-629.

[3] O. Arieli and A. Avron, The value of four values, Artificial Intelligence 102 (1998), pp. 97-141.

[4] N. D. Belnap. A useful four-valued logic, [in:] G. Epstein and J. M. Dunn (eds.), Modern Uses of Multiple-Valued Logic, Reidel, Dordrecht, 1977, pp. 7-37.

[5] J.-Y. Béziau, A new four-valued approach to modal logic, Logique et Analyse 54, 213 (2011), pp. 109-121.

[6] A. Białynicki-Birula and H. Rasiowa, On the representation of quasiBoolean algebras, Bulletin de la Academie Polonaise des Sciences, Cl. III, 5 (1957), pp. 259-261.

[7] R. Cignoli, Moisil algebras, Notas de Mathematica, No. 27, Universi-dad Nacional del Sur. Bahia Blanca, 1970.

[8] M. De and H. Omori, Classical negation and expansions of Belnap-Dunn logic, Studia Logica 103, 4 (2015), pp. 825-851.

[9] N. M. Ermolaeva and A. A. Mučnik, A modal extensions of Hao Wang-type of logical calculi, [in:] D. A. Bočvar (ed.), Investigations on Formalized Language and Non-Classical Logics, NAUKA Publishers, Moscow, 1974, pp. 172-193 (in Russian).

[10] N. M. Ermolaeva and A. A. Mučnik, Modal logics defined by endomorpismus in distributive lattices, [in:] D. A. Bočvar and V. N. Grishin (eds.), Investigations on the Set Theory and Non-classical Logics, NAUKA Publishers, Moscow, 1976, pp. 229-246 (in Russian).

[11] N. M. Ermolaeva and A. A. Mučnik, Functionally closed 4-valued extensions of Boolean algebras and the corresponding logics, [in:] A. I. Mihailov (ed.), Investigations on Non-classical Logics and the Set Theory, NAUKA Publishers, Moscow, 1979, pp. 298-315 (in Russian).

[12] M. Fitting, Bilattices and the theory of truth, Journal of Philosophical Logic 18 (1989), pp. 225-256.

[13] J. M. Font, Belnap's four-valued logic and De Morgan lattices, Logic Journal of the IGPL 5, 3 (1997), pp. 413-440. 
[14] M. L. Ginsberg, Multivalued logics: A uniform approach to inference in artificial intelligence, Computational Intelligence 4, 3 (1988), pp. 265-315.

[15] G. Iorgulescu, Connections between MVn algebras and n-valued Eukasiewicz-Moisil algebras Part I, Discrete Mathematics 181 (1998), pp. $155-177$.

[16] S. V. Jablonski, Functional constructions in k-valued logics, Studies of V. A. Steklov Mathematical Institute 51 (1958), pp. 5-142 (in Russian).

[17] A. S. Karpenko, Von Wright's truth logic and around, Logical Investigations 19 (2013), pp. 39-50.

[18] A. S. Karpenko, Lattices of four-valued modal logics, Logical Investigations 21, 1 (2015), pp. 122-137 (in Russian).

[19] D. Lau, Function Algebras on Finite Sets: A Basic Course on Many-Valued Logic and Clone Theory, Springer-Verlag, Berlin 2006.

[20] A. S. Karpenko and A. V. Chagrov, Modal propositional truth logic Tr and its completeness, Logical Investigations 22, 1 (2016), pp. 13-31 (in Russian).

[21] E. J. Lemmon, Algebraic semantics for modal logic I, The Journal of Symbolic Logic 31 (1966), pp. 46-65.

[22] C. I. Lewis and C. H. Langford, Symbolic Logic, N. Y., 1932 (2nd ed. in 1959).

[23] L. L. Maksimova, Interpolation theorems in modal logics and amalgamable varieties of topoboolean algebras, Algebra i Logica 18, 5 (1979), pp. 556-586 (in Russian).

[24] G. Malinowski, Many-Valued Logics, The Clarendon Press, Oxford 1993.

[25] H. Omori and K. Sano, Generalizing functional completeness in BelnapDunn logic, Studia Logica 103, 5 (2015), pp. 883-917.

[26] A. Pietz and U. Rivieccio, Nothing but the Truth, Journal of Philosophical Logic 42, 1 (2013), pp. 125-135.

[27] E. L Post, Introduction to a general theory of elementary propositions, American Journal of Mathematics 43, 3 (1921), pp. 163-185.

[28] A. P. Pynko, Functional completeness and axiomatizability within Belnap's four-valued logic and its expansion, Journal of Applied Non-Classical Logics 9, 1 (1999), pp. 61-105.

[29] J. B. Rosser and A. R. Turquette, Many-Valued Logics. North-Holland, Amsterdam 1952. 
[30] P. Ruet, Complete sets of connectives and complete sequent calculus for Belnap's logic, Tech. rep., Ecole Normal Superieure, 1966. Logic Colloquium 96. Document LIENS-96-28.

[31] K. Segerberg, On the logic of 'To-morrow', Theoria 33, 1 (1967), pp. $46-52$.

[32] B. Sobochiński, Modal system S4.4, Notre Dame Journal of Formal Logic 5, 4, (1964), pp. 305-312.

[33] B. Sobochiński, Certain extensions of modal system S4, Notre Dame Journal of Formal Logic 11, 3 (1970), pp. 347-367.

[34] R. Wójcicki and G. Malinowski (eds.) Selected Papers on Łukasiewicz Sentential Calculi. OSSOLINEUM, Wroclaw 1977.

[35] G. H. von Wright, Truth-logics, Logique et Analyse 30, 120 (1987), pp. 311-334 (repr. in Acta Philosopica Fennica 60 (1996), pp. 71-91).

Department of Logic

Institute of Philosophy

Russian Academy of Sciences

e-mail: as.karpenko@gmail.com 